\title{
PROJECTIONS INDUCING AUTOMORPHISMS OF STABLE UHF-ALGEBRAS
}

\author{
KAZUNORI KODAKA \\ coy Department of Mathematical Sciences, College of Science, Ryukyu University, Nishihara-cho, Okinawa, \\ 903-0213 Japan
}

(Received 24 October, 1997)

\begin{abstract}
Let $A$ be a UHF-algebra and $\mathbf{K}$ the $\mathrm{C}^{*}$-algebra of all compact operators on a countably infinite-dimensional Hilbert space. In this note we shall find all projections $p$ in $A$ with $p A p \cong A$ and, using these projections, we shall determine the group of automorphisms of $K_{0}(A \otimes \mathbf{K})$ induced by those of $A \otimes \mathbf{K}$ in some cases.
\end{abstract}

1991 Mathematics Subject Classification 46 L40.

0. Introduction. Let $A$ be a UHF-algebra and $\mathbf{K}$ the $\mathrm{C}^{*}$-algebra of all compact operators on a countably infinite-dimensional Hilbert space. Let $p$ be a projection in $A \otimes \mathbf{K}$ with $p(A \otimes \mathbf{K}) p \cong A$. In [9] we showed that we can construct any automorphism of $A \otimes \mathbf{K}$ using the projection $p$ above, an automorphism of $A$ and a unitary element in $M(A \otimes \mathbf{K})$, where $M(A \otimes \mathbf{K})$ is the multiplier algebra of $A \otimes \mathbf{K}$. But since $A$ is a UHF-algebra, it suffices to find all projections $p$ in $A$ with $p A p \cong A$ in order to determine the group of automorphisms of $K_{0}(A \otimes \mathbf{K})$ induced by those of $A \otimes \mathbf{K}$. By the above result we can compute the Picard group of $A$ in some cases. Furthermore let $\beta$ be an automorphism of $A \otimes \mathbf{K}$ with $\beta_{*} \neq$ id on $K_{0}(A \otimes \mathbf{K})$. Then, by Rørdam [12] a crossed product $A \otimes \mathbf{K} \times{ }_{\beta} \mathbf{Z}$ is a purely infinite simple $\mathrm{C}^{*}$-algebra and its isomorphism class can be determined by Elliott, Evans and Kishimoto [5] if the automorphism $\beta_{*}$ of $K_{0}(A \otimes \mathbf{K})$ is known to us.

Since $A \otimes \mathbf{K}$ is an AF-algebra, we can determine the group of automorphisms of $K_{0}(A \otimes \mathbf{K})$ induced by those of $A \otimes \mathbf{K}$ by Blackadar [2, Theorem 7.3.2]. In fact if $A=M_{2 \infty}$, we can easily do it, where $M_{2 \infty}$ is the UHF-algebra of type $2^{\infty}$. However, it seems difficult in general to determine order-preserving automorphisms of the dimension group $K_{0}(A \otimes \mathbf{K})$ and so we apply the method above to determine projections $p$ in $A$ with $p A p \cong A$.

1. Preliminaries. For each $n \in \mathbf{N}$, let $M_{n}$ be the $\mathrm{C}^{*}$-algebra of $n \times n$-matrices over C. For positive integers $m(1), m(2) \geq 2$ let $l$ be a monomorphism of $M_{m(1)}$ into $M_{m(1) m(2)}$ such that $\imath\left(I_{m(1)}\right)=I_{m(1) m(2)}$, where $I_{m(1)}$ and $I_{m(1) m(2)}$ are the unit elements in $M_{m(1)}$ and $M_{m(1) m(2)}$ respectively. Given a sequence $\{m(n)\}_{n=1}^{\infty}$ of positive integers greater than 1 , let $m(n) !=\prod_{k=1}^{n} m(k)$. We consider the inductive system

$$
M_{m(1) !} \stackrel{l}{\longrightarrow} M_{m(2) !} \stackrel{l}{\longrightarrow} \cdots \stackrel{l}{\longrightarrow} M_{m(n) !} \stackrel{l}{\longrightarrow} \cdots .
$$

We call the $\mathrm{C}^{*}$-algebra generated by the inductive system above a UHF-algebra of type $\{m(n) !\}$.

Let $A$ be a UHF-algebra and $\tau$ the unique tracial state on $A$. Then by Blackadar [2], $K_{0}(A)$ is a simple dimension group which is a dense subgroup of $\mathbf{Q}$ containing $\mathbf{Z}$. 
Let $\tau_{*}$ be the homomorphism of $K_{0}(A)$ to $\mathbf{R}$ induced by $\tau$. By Blackadar [1, Theorem 3.9] $\tau_{*}$ is injective and the positive cone of $K_{0}(A)$ is given by the formula

$$
K_{0}(A)_{+}=\left\{x \in K_{0}(A) \mid \tau_{*}(x) \geq 0\right\} .
$$

We identify $K_{0}(A)$ with $\tau_{*}\left(K_{0}(A)\right)$. Since $K_{0}(A)$ is a dense subgroup of $\mathbf{Q}$, an automorphism of $K_{0}(A)$ is multiplication by a positive rational number.

Lemma 1.1. For any automorphism $\alpha$ of $A, \alpha_{*}=\mathrm{id}$ on $K_{0}(A)$.

Proof. This can easily be proved using the facts that, by the uniqueness of trace, $\alpha$ preserves the trace $\tau$ and the homomorphism $\tau_{*}: K_{0}(A) \rightarrow \mathbf{R}$ is injective. Q.E.D.

Let $\mathbf{K}$ be the $\mathrm{C}^{*}$-algebra of all compact operators on a countably infinitedimensional Hilbert space and $\left\{e_{i j}\right\}_{i, j \in \mathbf{Z}}$ matrix units of $\mathbf{K}$. Let $\operatorname{Tr}$ be the canonical trace on $\mathbf{K}$. Then $\tau \otimes \operatorname{Tr}$ is a densely defined lower semi-continuous trace on $A \otimes \mathbf{K}$ and, as described in Elliott, Evans and Kishimoto [5], it is unique up to a constant multiple. Let $\beta$ be an automorphism of $A \otimes \mathbf{K}$. We define $s(\beta) \in \mathbf{Q}$ by $(\tau \otimes \mathrm{Tr}) \circ \beta=s(\beta)(\tau \otimes \mathrm{Tr})$. Then an automorphism $\beta_{*}$ of $K_{0}(A \otimes \mathbf{K})$ is multiplication by the positive rational number $s(\beta)$.

Let $M_{n}(A)$ be the $\mathrm{C}^{*}$-algebra of $n \times n$-matrices over $A$, for any $n \in \mathbf{N}$; we identify $M_{n}(A)$ with $A \otimes M_{n}$. Let $p$ be a projection in $\cup_{n=1}^{\infty} M_{n}(A) \subset A \otimes \mathbf{K}$ with $p(A \otimes \mathbf{K}) p \cong A$. We denote by $\chi_{p}$ an isomorphism of $A$ onto $p(A \otimes \mathbf{K}) p$. By Brown [3, Lemma 2.5], there is a partial isometry $z \in M(A \otimes \mathbf{K} \otimes \mathbf{K})$ such that $z^{*} z=p \otimes 1$ and $z z^{*}=1 \otimes 1 \otimes 1$. Let $\psi$ be an isomorphism of $\mathbf{K} \otimes \mathbf{K}$ onto $\mathbf{K}$ with $\psi_{*}=$ id of $K_{0}(\mathbf{K} \otimes \mathbf{K})$ onto $K_{0}(\mathbf{K})$. Let $\beta_{p}$ be the automorphism of $A \otimes \mathbf{K}$ defined by

$$
\beta_{p}=(\mathrm{id} \otimes \psi) \circ \operatorname{Ad}(z) \circ\left(\chi_{p} \otimes \mathrm{id}\right) .
$$

Lemma 1.2. With the notations above the automorphism $\beta_{p *}$ of $K_{0}(A \otimes \mathbf{K})$ is multiplication by $(\tau \otimes \operatorname{Tr})(p)$.

Proof. It suffices to show that $s\left(\beta_{p}\right)=(\tau \otimes \operatorname{Tr})(p)$. Let $(\tau \otimes \operatorname{Tr})_{*}$ be the homomorphism of $K_{0}(A \otimes \mathbf{K})$ to $\mathbf{R}$ induced by $\tau \otimes \operatorname{Tr}$. We note that $\beta_{p}\left(1 \otimes e_{00}\right)$ is in the ideal of definition of $\tau \otimes \operatorname{Tr}$ by [7, Lemma 1]. Hence

$$
\begin{aligned}
(\tau \otimes \mathrm{Tr}) \circ \beta_{p}\left(1 \otimes e_{00}\right) & =(\tau \otimes \operatorname{Tr})_{*} \circ \beta_{p *}\left(\left[1 \otimes e_{00}\right]\right) \\
& =(\tau \otimes \operatorname{Tr})_{*} \circ(\mathrm{id} \otimes \psi)_{*}\left(\left[z\left(p \otimes e_{00}\right) z^{*}\right]\right) \\
& =(\tau \otimes \operatorname{Tr})_{*} \circ(\mathrm{id} \otimes \psi)_{*}\left(\left[p \otimes e_{00}\right]\right) \\
& =(\tau \otimes \operatorname{Tr})(p) .
\end{aligned}
$$

Since $(\tau \otimes \operatorname{Tr}) \circ \beta_{p}=s\left(\beta_{p}\right)(\tau \otimes \operatorname{Tr}) \quad$ and $\quad(\tau \otimes \operatorname{Tr})\left(1 \otimes e_{00}\right)=1 \quad$ it follows that $s\left(\beta_{p}\right)=(\tau \otimes \operatorname{Tr})(p)$. Q.E.D.

Corollary 1.3. Let $\beta_{p}$ be as above. If $(\tau \otimes \operatorname{Tr})(p)>1$, there is a projection $q \in A$ with $q A q \cong A$ such that $\beta_{p *}^{-1}=\beta_{q \otimes e_{00 *}}$ on $K_{0}(A \otimes \mathbf{K})$.

Proof. By [9, Theorem 4.5 and Remark 2.1], there are an $n \in \mathbf{N}$, a projection $q_{1} \in M_{n}(A)$, an automorphism $\alpha$ of $A$ and a unitary element $w \in M(A \otimes \mathbf{K})$ such that 


$$
q_{1}(A \otimes \mathbf{K}) q_{1} \cong A, \quad \beta_{p}^{-1}=\operatorname{Ad}(w) \circ \beta_{q_{1}} \circ(\alpha \otimes \mathrm{id}),
$$

where $M(A \otimes \mathbf{K})$ is the multiplier algebra of $A \otimes \mathbf{K}$. By Lemma 1.1 and [9, Lemma 1.1] $\beta_{p *}^{-1}=\beta_{q_{1} *}$. Hence, by Lemma 1.2, $(\tau \otimes \operatorname{Tr})\left(q_{1}\right)(\tau \otimes \operatorname{Tr})(p)=1$. We note that $\tau(\operatorname{Proj} A)=\tau_{*}\left(K_{0}(A)\right) \cap[0,1]$, where $\operatorname{Proj} A$ is the set of all projections in $A$. Since $A$ has cancellation, there is a projection $q \in A$ such that $q \otimes e_{00}$ is unitarily equivalent to $q_{1}$ in $(A \otimes \mathbf{K})^{+}$, where $(A \otimes \mathbf{K})^{+}$is the unitized $\mathrm{C}^{*}$-algebra of $A \otimes \mathbf{K}$. Thus $q A q \cong A$ and $\beta_{p *}^{-1}=\beta_{q \otimes e_{00} *}$ on $K_{0}(A \otimes \mathbf{K})$. Q.E.D.

Let $\operatorname{Aut}\left(K_{0}(A \otimes \mathbf{K})\right)$ be the group of automorphisms of $K_{0}(A \otimes \mathbf{K})$ and let

$$
S=\left\{\beta_{p \otimes e_{00 *}} \in \operatorname{Aut}\left(K_{0}(A \otimes \mathbf{K})\right) \mid p \text { is a projection in } A \text { with } p A p \cong A\right\} .
$$

COROLlary 1.4. With the notations above, $S$ is a semigroup of automorphisms of $K_{0}(A \otimes \boldsymbol{K})$ with the unit element.

Proof. Since $S$ is a subset of the group $\operatorname{Aut}\left(K_{0}(A \otimes \mathbf{K})\right)$, it suffices to show that $S$ is invariant under the product of $\operatorname{Aut}\left(K_{0}(A \otimes \mathbf{K})\right)$ and that $S$ has the unit element in $\operatorname{Aut}\left(K_{0}(A \otimes \mathbf{K})\right)$. Since $\tau(1)=1, \beta_{1 \otimes e_{00} *}$ is the unit element in $\operatorname{Aut}\left(K_{0}(A \otimes \mathbf{K})\right)$. Thus $S$ has the unit element in $\operatorname{Aut}\left(K_{0}(A \otimes \mathbf{K})\right)$. For $j=1,2$, let $p_{j}$ be a projection in $A$ with $p_{j} A p_{j} \cong A$. Then, in the same way as in the proof of Corollary 1.3 , we see that there is a projection $p_{3}$ in $A$ such that

$$
\tau\left(p_{3}\right)=\tau\left(p_{1}\right) \tau\left(p_{2}\right), \quad p_{3} A p_{3} \cong A .
$$

Since $\tau\left(p_{3}\right)=\tau\left(p_{1}\right) \tau\left(p_{2}\right)$, by Lemma 1.2 we deduce that $\beta_{p_{3} *}=\beta_{p_{1 *}} \circ \beta_{p_{2} *}$. Hence $\beta_{p_{1 *}} \circ \beta_{p_{2 *}} \in S$. Therefore we obtain the conclusion. Q.E.D.

Remark 1.5. Let $A$ be a UHF-algebra of type $\{m(n)$ ! $\}$. By Corollary 1.3 and [9], the group of automorphisms of $K_{0}(A \otimes \mathbf{K})$ induced by those of $A \otimes \mathbf{K}$ is generated by $S$ and, since an automorphism of $K_{0}(A \otimes \mathbf{K})$ is multiplication by a positive rational number, by Lemma 1.2 and Corollary 1.3 we have

$$
S=\{\tau(p) \in \mathbf{Q} \mid p \text { is a projection in } A \text { with } p A p \cong A\} .
$$

Furthermore, by Blackadar [2, Proposition 4.6.6],

$$
S=\left\{\tau(p) \in \mathbf{Q} \mid p \text { is a projection in } \cup_{n=1}^{\infty} M_{m(n)} \text { ! with } p A p \cong A\right\} .
$$

2. Projections $\boldsymbol{p}$ in $\boldsymbol{A}$ with $\boldsymbol{p} \boldsymbol{A p}$ isomorphic to $\boldsymbol{A}$. Let $A$ be a UHF-algebra of type $\{m(n) !\}$. Following Glimm [6] we define a function $f(\{m(n) !\})$ whose domain is the prime numbers. For each prime number $r$, let

$$
f\left(\{m(n) !\}(r)=\sup \left\{k \in \mathbf{N} \mid \text { there is an } n \in \mathbf{N} \text { such that } r^{k} \text { divides } m(n) !\right\} .\right.
$$

Also, for each subset $N$ of $\mathbf{N}$ we denote by $\#(N)$ the number of elements in $N$.

Lemma 2.1. Let $f(\{m(n) !\})$ be as above and $r$ a prime number. Then the following conditions hold: 
(1) $f(\{m(n) !\})(r)=\infty$ if and only if $\#\{n \in N \mid r$ divides $m(n)\}=\infty$,

(2) $f(\{m(n) !\})(r)=0$ if and only if $r$ does not divide $m(n)$ for any $n \in N$,

(3) $f(\{m(n) !\})(r)<\infty$ if and only if there is an $n_{0} \in N$ such that $r$ does not divide $m(n)$ for any $n \geq n_{0}$.

Proof. (1) $\Rightarrow$ : We suppose that $\#\{n \in \mathbf{N} \mid r$ divides $m(n)\}<\infty$. Then there is an $n_{0} \in \mathbf{N}$ such that $r$ does not divide $m(n)$ for any $n \geq n_{0}$. Thus

$$
\begin{aligned}
f(\{m(n) !\})(r)= & \sup \left\{k \in \mathbf{N} \mid \text { there is an } n \in \mathbf{N} \text { such that } r^{k} \text { divides } m(n) !\right\} \\
= & \sup \left\{k \in \mathbf{N} \mid \text { there is an integer } n \text { with } 1 \leq n \leq n_{0}-1 \text { such that } r^{k}\right. \\
& \text { divides } m(n) !\} \\
< & \infty .
\end{aligned}
$$

This is a contradiction. Therefore $\#\{n \in \mathbf{N} \mid r$ divides $m(n)\}=\infty$.

$\Leftarrow$ : For any $k \in \mathbf{N}$ there is a set $\left\{n_{1}, n_{2}, \ldots, n_{k}\right\} \subset\{n \in \mathbf{N} \mid r$ divides $m(n)\}$ with $n_{1}<n_{2}<\ldots<n_{k}$. Since $r$ divides $m\left(n_{j}\right)$, for $j=1,2, \ldots, k, r^{k}$ divides $m\left(n_{k}\right)$ !. Thus $f(\{m(n) !\}(r) \geq k$. Since $k$ is an arbitrary positive integer, $f(\{m(n) !\})(r)=\infty$.

(2) $\Rightarrow$ : If there is an $n_{0} \in \mathbf{N}$ such that $r$ divides $m\left(n_{0}\right)$, then $r$ divides $m\left(n_{0}\right)$ !. Hence $f(\{m(n) !\})(r) \geq 1$. This is a contradiction. Thus $r$ does not divide $m(n)$, for any $n \in \mathbf{N}$.

$\Leftarrow$ : If $f(\{m(n) !\})(r) \geq 1$, then there is an $n_{0} \in \mathbf{N}$ such that $r$ divides $m\left(n_{0}\right)$ !. Hence there is an $n_{1} \in \mathbf{N}$ such that $r$ divides $m\left(n_{1}\right)$. This is a contradiction. Thus $f(\{m(n) !\})(r)=0$.

(3) is equivalent to (1). Q.E.D.

Let $A$ be a UHF-algebra of type $\{m(n) !\}$. We suppose that $f(\{m(n) !\})(r)=0$ or $\infty$, for any prime number $r$. If $f(\{m(n) !\})(r)=0$, for any prime number $r$, then $A \cong \mathbf{C}$ and so we also suppose that

$\#\{r \mid r$ is a prime number with $f(\{m(n) !\})(r)=\infty\} \geq 1$.

Lemma 2.2. With the notations and assumptions above, let $n_{0}$ be a positive integer and $p$ a projection in $M_{m\left(n_{0}\right) !}$ with $\tau(p)=\frac{k}{m\left(n_{0}\right) !}$. Then the following conditions hold.

(1) If $k=1$, then $p A p \cong A$,

We suppose that $k \neq 1$. Let $k=c_{1}^{d_{1}} \ldots c_{h}^{d_{h}}$ be the decomposition of $k$ by prime factors with $d_{j} \neq 0$ for $j=1,2, \ldots, h$.

(2) If $f(\{m(n) !\})\left(c_{j}\right)=\infty$, for $j=1,2, \ldots, h$, then $p A p \cong A$.

(3) If there is an integer $j_{0}$ with $1 \leq j_{0} \leq h$ such that $f(\{m(n) !\})\left(c_{j_{0}}\right)=0$, then $p A p$ is not isomorphic to $A$.

Proof. (1) For the UHF-algebra $p A p$ we have the inductive system

$$
M_{m\left(n_{0}+1\right)} \longrightarrow M_{m\left(n_{0}+1\right) m\left(n_{0}+2\right)} \longrightarrow \ldots \longrightarrow M_{m\left(n_{0}+1\right) \ldots m\left(n_{0}+n\right)} \longrightarrow \cdots
$$

For any prime number $r$ with $f(\{m(n) !\})(r)=\infty$, $\#\{n \in \mathbf{N} \mid r$ divides $m(n)\}=\infty$, by Lemma 2.1. Hence $f\left(\left\{m\left(n_{0}+1\right) \ldots m\left(n_{0}+n\right)\right\}\right)(r)=\infty$. Also, for any prime number $r$ with $f(\{m(n) !\})(r)=0, \#\{n \in \mathbf{N} \mid r$ divides $m(n)\}=0$, by Lemma 2.1. Hence $f\left(\left\{m\left(n_{0}+1\right) \ldots m\left(n_{0}+n\right)\right\}\right)(r)=0$. Thus 


$$
f(\{m(n) !\})=f\left(\left\{m\left(n_{0}+1\right) \ldots m\left(n_{0}+n\right)\right\}\right) .
$$

Therefore, by Glimm [6, Theorem 1.12], we have $p A p \cong A$.

(2) For the UHF-algebra $p A p$ we have the inductive system

$$
M_{k} \longrightarrow M_{k m\left(n_{0}+1\right)} \longrightarrow \ldots \longrightarrow M_{k m\left(n_{0}+1\right) \ldots m\left(n_{0}+n-1\right)} \longrightarrow \cdots
$$

For any prime number $r$ with $f(\{m(n) !\})(r)=\infty$, we have

$$
f\left(\left\{k m\left(n_{0}+1\right) \ldots m\left(n_{0}+n-1\right)\right\}\right)(r)=\infty,
$$

by Lemma 2.1. For any prime number $r$ with $f(\{m(n) !\})(r)=0$, we have $f\left(\left\{k m\left(n_{0}+1\right) \ldots m\left(n_{0}+n-1\right)\right\}\right)(r)=0$ since $r$ does not divide $k$ and $m(n)$ for any $n \in \mathbf{N}$. Therefore by Glimm [6, Theorem 1.12] $p A p \cong A$.

(3) Since $c_{j_{0}}$ divides $k$ and does not divide $m(n)$, for any $n \in \mathbf{N}$,

$$
f\left(\left\{k m\left(n_{0}+1\right) \ldots m\left(n_{0}+n-1\right)\right\}\right)\left(c_{j_{0}}\right)=d_{j_{0}} \geq 1 .
$$

On the other hand $f(\{m(n) !\})\left(c_{j_{0}}\right)=0$. Hence by Glimm [6, Theorem 1.12] $p A p$ is not isomorphic to $A$. Q.E.D.

THEOREM 2.3. With the same assumptions as in Lemma 2.2, let $n_{0}$ be a positive integer and $p$ a projection in $M_{m\left(n_{0}\right) !}$ with $\tau(p)=\frac{k}{m\left(n_{0}\right) !}$. Then $p A p \cong A$ if and only if $k=1$ or $k=c_{1}^{d_{1}} \ldots c_{h}^{d_{h}}$ with $f(\{m(n) !\})\left(c_{j}\right)=\infty$ and $d_{j} \neq 0$, for $j=1,2, \ldots, h$.

Proof. This is immediate, by Lemma 2.2. Q.E.D.

Let $A$ be a UHF-algebra of type $\{m(n) !\}$. We suppose that

$$
1 \leq \#\{r \mid r \text { is a prime number with } 1 \leq f(\{m(n) !\})(r)<\infty\}<\infty,
$$

$\#\{r \mid r$ is a prime number with $f(\{m(n) !\})(r)=\infty\}=\infty$.

Let $\left\{r_{j}\right\}_{j=1}^{l}$ be the set of all prime numbers with $1 \leq f(\{m(n) !\})\left(r_{j}\right)<\infty$. We put $t_{j}=f(\{m(n) !\})\left(r_{j}\right)$, for $j=1,2, \ldots, l$. By the assumptions above there is an $n_{0} \in \mathbf{N}$ such that $r_{1}^{t_{1}} \ldots r_{l}^{t_{l}}$ divides $m\left(n_{0}\right)$ ! and, for any $n \geq n_{0}$ and $j=1,2, \ldots, l, r_{j}$ does not divide $m(n)$. Let $n_{1}$ be any positive integer with $n_{1} \geq n_{0}$ and $p$ a projection in $M_{m\left(n_{1}\right) \text { ! }}$ with $\tau(p)=\frac{k}{m\left(n_{1}\right) !}$. We note that, for the UHF-algebra $p A p$, we have the inductive system

$$
M_{k} \longrightarrow M_{k m\left(n_{0}+1\right)} \longrightarrow \ldots \longrightarrow M_{k m\left(n_{0}+1\right) \ldots m\left(n_{0}+n-1\right)} \longrightarrow \cdots
$$

Lemma 2.4. With the notations and assumptions above, the following conditions hold.

(1) If $k=r_{1}^{t_{1}} \ldots r_{l}^{t_{l}}$, then $p A p \cong A$,

(2) If $r_{1}^{t_{1}} \ldots r_{l}^{t_{l}}$ does not divide $k$, then $p$ Ap is not isomorphic to $A$.

Proof. (1) Since $f(\{m(n) !\})\left(r_{j}\right)=t_{j}$, for $j=1,2, \ldots, l$, and $r_{j}$ does not divide $m(n)$ for any $n \geq n_{1}$ and $j=1,2, \ldots, l$, we have 


$$
f\left(\left\{k m\left(n_{1}+1\right) \ldots m\left(n_{1}+n-1\right)\right\}\right)\left(r_{j}\right)=t_{j} .
$$

Also, by Lemma 2.1,

$$
\begin{array}{cl}
f\left(\left\{k m\left(n_{1}+1\right) \ldots m\left(n_{1}+n-1\right)\right\}\right)(r)=\infty, & \text { if } f(\{m(n) !\})(r)=\infty, \\
f\left(\left\{k m\left(n_{1}+1\right) \ldots m\left(n_{1}+n-1\right)\right\}\right)(r)=0, & \text { if } f(\{m(n) !\})(r)=0 .
\end{array}
$$

Hence, by Glimm [6, Theorem 1.12], $p A p \cong A$.

(2) Since $r_{1}^{t_{1}} \ldots r_{l}^{t_{l}}$ does not divide $k$, there is a $j_{0} \in \mathbf{N}$ with $1 \leq j_{0} \leq l$ such that $r_{j_{0}}^{t_{j_{0}}}$ does not divide $k$. Hence

$$
\left.f\left(\left\{k m\left(n_{1}+1\right) \ldots m\left(n_{1}+n-1\right)\right\}\right)\left(r_{j_{0}}\right)<t_{j_{0}}=f(\{m(n) !\})\right)\left(r_{j_{0}}\right) .
$$

Thus $p A p$ is not isomorphic to $A$ by Glimm [6, Theorem 1.12]. Q.E.D.

By Lemma 2.4 (2), if $p A p \cong A$, there is a $k_{1} \in \mathbf{N}$ such that $k=r_{1}^{t_{1}} \ldots r_{l}^{t_{l}} k_{1}$.

LEMMA 2.5. With the same notations as in Lemma 2.4, we suppose that there is a $k_{1} \in N$ such that $k=r_{1}^{t_{1}} \ldots r_{l}^{t_{l}} k_{1}$. Let $k_{1}=c_{1}^{d_{1}} \ldots c_{h}^{d_{h}}$ be the decomposition of $k_{1}$ by prime factors with $d_{j} \neq 0$, for $j=1,2, \ldots, h$. Then the following conditions hold.

(1) If there is a $j_{0} \in \mathbf{N}$ with $1 \leq j_{0} \leq h$ such that $f(\{m(n) !\})\left(c_{j_{0}}\right)=0$, then $p A p$ is not isomorphic to $A$.

(2) If $f(\{m(n) !\})\left(c_{j}\right)=\infty$ for $j=1,2, \ldots, h$, then $p A p \cong A$.

Proof. (1) Since $f\left(\left\{k m\left(n_{1}+1\right) \ldots m\left(n_{1}+n-1\right)\right\}\right)\left(c_{j_{0}}\right) \geq 1$, we have

$$
f\left(\left\{k m\left(n_{1}+1\right) \ldots\left(n_{1}+n-1\right)\right\}\right) \neq f(\{m(n) !\}) .
$$

Thus $p A p$ is not isomorphic to $A$, by Glimm [6, Theorem 1.12].

(2) By Lemma 2.1, for any prime number $r$ with $f(\{m(n) !\})(r)=\infty$, we have

$$
f\left(\left\{k m\left(n_{1}+1\right) \ldots m\left(n_{1}+n-1\right)\right\}\right)(r)=\infty .
$$

Let $r$ be a prime number with $1 \leq f(\{m(n) !\})(r)<\infty$. Then there is a $j_{0} \in \mathbf{N}$ with $1 \leq j_{0} \leq l$ such that $r=r_{j_{0}}$ and that $f(\{m(n) !\})(r)=t_{j_{0}}$. Since $r_{j_{0}}^{t_{0}}$ divides $k$ and $r_{j_{0}}$ does not divide $m(n)$, for any $n \geq n_{1}, f\left(\left\{k m\left(n_{1}+1\right) \ldots m\left(n_{1}+n-1\right)\right\}\right)(r)=t_{j_{0}}$. Let $r$ be a prime number with $f(\{m(n) !\})(r)=0$. Then $r \neq r_{j}$, for $j=1,2, \ldots, l$, and $r \neq c_{j}$, for $j=1,2, \ldots, h$, since $f(\{m(n) !\})\left(c_{j}\right)=\infty$, for $j=1,2, \ldots, h$. Hence $r$ does not divide $k$. Hence

$$
f\left(\left\{k m\left(n_{1}+1\right) \ldots m\left(n_{1}+n-1\right)\right\}\right)(r)=0 .
$$

Thus $p A p \cong A$, by Glimm [6, Theorem 1.12]. Q.E.D.

THEOREM 2.6. With the notations and assumptions above, let $n_{1}$ be an integer with

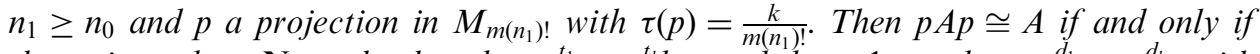
there is a $k_{1} \in \mathbf{N}$ such that $k=r_{1}^{t_{1}} \ldots r_{l}^{t_{l}} k_{1}$ and $k_{1}=1$ or $k_{1}=c_{1}^{d_{1}} \ldots c_{h}^{d_{h}}$ with $f(\{m(n) !\})\left(c_{j}\right)=\infty$ and $d_{j} \neq 0$ for $j=1,2, \ldots, h$.

Proof. This is immediate by Lemmas 2.4 and 2.5 Q.E.D. 
Let $A$ be a UHF-algebra of type $\{m(n) !\}$. We suppose that

$\#\{r \mid r$ is a prime number with $1 \leq f(\{m(n) !\})(r)<\infty\}=\infty$,

$\#\{r \mid r$ is a prime number with $f(\{m(n) !\})(r)=\infty\} \geq 1$.

By the assumptions above we may assume that, for any $n \in \mathbf{N}, m(n)$ ! has a prime number $r$ as a factor with $1 \leq f(\{m(n) !\})(r)<\infty$. Let $p$ be a projection in $M_{m\left(n_{0}\right) \text { ! with }}$ $\tau(p)=\frac{k}{m\left(n_{0}\right) !}$. For the UHF-algebra $p A p$ we have the inductive system

$$
M_{k} \longrightarrow M_{k m\left(n_{0}+1\right)} \longrightarrow \ldots \longrightarrow M_{k m\left(n_{0}+1\right) \ldots m\left(n_{0}+n-1\right)} \longrightarrow \cdots
$$

By Lemma 2.1 we can easily see that

$$
f\left(\left\{k m\left(n_{0}+1\right) \ldots m\left(n_{0}+n-1\right)\right\}\right)(r)=\infty,
$$

for any prime number $r$ with $f(\{m(n) !\})(r)=\infty$. Let $r_{1}^{s_{1}} \ldots r_{l}^{s_{l}}$ be a factor of $m\left(n_{0}\right)$ ! with $1 \leq f(\{m(n) !\})\left(r_{j}\right)=t_{j}<\infty$ and $1 \leq s_{j} \leq t_{j}$, for $j=1,2, \ldots, l$, such that $r_{j}$ does not divide $\frac{m\left(n_{0}\right) !}{r_{1}^{1} \ldots r_{l}^{l}}$, for $j=1,2, \ldots, l$, and $r$ does not divide $m\left(n_{0}\right) !$, for any prime number $r$ with $r \neq r_{j}$ for $j=1,2, \ldots, l$ and $1 \leq f(\{m(n) !\})(r)<\infty$.

LEMma 2.7. With the notations and assumptions above, if $r_{1}^{s_{1}} \ldots r_{l}^{s_{l}}$ does not divide $k$, then $\mathrm{pAp}$ is not isomorphic to $A$.

Proof. Since $f(\{m(n) !\})\left(r_{j}\right)=t_{j}$, for $j=1,2, \ldots, l$, there is an $n_{j} \in \mathbf{N}$ with $n_{j} \geq n_{0}+1$ such that $r_{j}^{t_{j}-s_{j}}$ divides $\frac{m\left(n_{j}\right) !}{m\left(n_{0}\right) !}$. Since $r_{1}^{s_{1}} \ldots r_{l}^{s_{l}}$ does not divide $k$, there is a $j_{0} \in \mathbf{N}$ with $1 \leq j_{0} \leq l$ such that $r_{j_{0}}^{s_{0}}$ does not divide $k$. Thus

$$
f\left(\left\{k m\left(n_{0}+1\right) \ldots m\left(n_{0}+n-1\right)\right\}\right)\left(r_{j_{0}}\right)<s_{j_{0}}+\left(t_{j_{0}}-s_{j_{0}}\right)=t_{j_{0}} .
$$

On the other hand $f(\{m(n) !\})\left(r_{j_{0}}\right)=t_{j_{0}}$. Thus $p A p$ is not isomorphic to $A$ by Glimm [6, Theorem 1.12]. Q.E.D.

By Lemma 2.7, if $p A p \cong A$, then there is a $k_{1} \in \mathbf{N}$ such that $k=r_{1}^{s_{1}} \ldots r_{l}^{s_{l}} k_{1}$. So we suppose that there is a $k_{1} \in \mathbf{N}$ such that $k=r_{1}^{s_{1}} \ldots r_{l}^{s_{l}} k_{1}$.

LEMMA 2.8. With the notations and assumptions above, if there is a prime number $r_{0}$ with $f(\{m(n) !\})\left(r_{0}\right)<\infty$ such that $r_{0}$ divides $k_{1}$, then $p$ Ap is not isomorphic to $A$.

Proof. If $f(\{m(n) !\})\left(r_{0}\right)=0$, then $f\left(\left\{k m\left(n_{0}+1\right) \ldots m\left(n_{0}+n-1\right)\right\}\right)\left(r_{0}\right) \geq 1$, since $r_{0}$ divides $k$. Thus $p A p$ is not isomorphic to $A$ by Glimm [6, Theorem 1.12]. We suppose that $f(\{m(n) !\})\left(r_{0}\right) \geq 1$. Furthermore, we suppose that there is a $j_{0} \in \mathbf{N}$ with $1 \leq j_{0} \leq l$ such that $r_{0}=r_{j_{0}}$. If $s_{j_{0}}=t_{j_{0}}$, then $r_{j_{0}}^{t_{0}+1}$ divides $k$, since

$$
k=r_{1}^{s_{1}} \ldots r_{j_{0}}^{t_{j_{0}}+1} \ldots r_{l}^{s_{l}} \frac{k_{1}}{r_{j_{0}}} .
$$

Hence

$$
f\left(\left\{k m\left(n_{0}+1\right) \ldots m\left(n_{0}+n-1\right)\right\}\right)\left(r_{j_{0}}\right)=t_{j_{0}}+1 .
$$


Since $f(\{m(n) !\})\left(r_{j_{0}}\right)=t_{j_{0}}, p A p$ is not isomorphic to $A$, by Glimm [6, Theorem 1.12]. If $s_{j_{0}}<t_{j_{0}}$, then $r_{j_{0}}^{s_{j_{0}}+1}$ divides $k$, since

$$
k=r_{1}^{s_{1}} \ldots r_{j_{0}}^{s_{j_{0}}+1} \ldots r_{l}^{s_{l}} \frac{k_{1}}{r_{j_{0}}} .
$$

Furthermore, since $f(\{m(n) !\})\left(r_{j_{0}}\right)=t_{j_{0}}$, there is an $n_{j_{0}} \in \mathbf{N}$ with $n_{j_{0}} \geq n_{0}+1$ such that $r^{t_{j_{0}}-s_{j_{0}}}$ divides $\frac{m\left(n_{j_{0}}\right) !}{m\left(n_{0}\right) !}$. Thus

$$
f\left(\left\{k m\left(n_{0}+1\right) \ldots m\left(n_{0}+n-1\right)\right\}\right)\left(r_{j_{0}}\right) \geq s_{j_{0}}+1+t_{j_{0}}-s_{j_{0}}=t_{j_{0}}+1 .
$$

Hence, by Glimm [6, Theorem 1.12], $p A p$ is not isomorphic to $A$.

Next, we suppose that $r_{0} \neq r_{j}$, for $j=1,2, \ldots, l$. Then, since $r_{0}$ does not divide $m\left(n_{0}\right)$ !, we have

$$
f\left(\left\{k m\left(n_{0}+1\right) \ldots m\left(n_{0}+n-1\right)\right\}\right)\left(r_{0}\right)=1+f(\{m(n) !\})\left(r_{0}\right) .
$$

Thus $p A p$ is not isomorphic to $A$, by Glimm [6, Theorem 1.12]. Q.E.D.

By Lemmas 2.7 and 2.8, if $p A p \cong A$, then there is a $k_{1} \in \mathbf{N}$ such that $k=r_{1}^{s_{1}} \ldots r_{l}^{s_{l}} k_{1}$ and $k_{1}=1$ or $k_{1}=c_{1}^{d_{1}} \ldots c_{h}^{d_{h}}$, where $c_{j}$ is a prime number with $f(\{m(n) !\})\left(c_{j}\right)=\infty$ and $d_{j} \neq 0$ for $j=1,2, \ldots, h$.

Lemma 2.9. With the same assumptions as in Lemma 2.8, we suppose that there is a $k_{1} \in \mathbf{N}$ such that $k=r_{1}^{s_{1}} \ldots r_{l}^{s_{l}} k_{1}$ and $k_{1}=1$ or $k_{1}=c_{1}^{d_{1}} \ldots c_{h}^{d_{h}}$, where $c_{j}$ is a prime number with $f(\{m(n) !\})\left(c_{j}\right)=\infty$ and $d_{j} \neq 0$ for $j=1,2, \ldots, h$. Then $p A p \cong A$.

Proof. We suppose that $r$ is a prime number such that $r=r_{j_{0}}$, for some $j_{0} \in \mathbf{N}$, with $1 \leq j_{0} \leq l$. Then, since $f(\{m(n) !\})\left(r_{j_{0}}\right)=t_{j_{0}}$, there is an $n_{j_{0}} \in \mathbf{N}$ with $n_{j_{0}} \geq n_{0}+1$ such that $r^{t_{j_{0}}-s_{j_{0}}}$ divides $\frac{m\left(n_{j_{0}}\right) !}{m\left(n_{0}\right) !}$. Since $r_{j_{0}}$ does not divide $k_{1}$, we have

$$
f\left(\left\{k m\left(n_{0}+1\right) \ldots m\left(n_{0}+n-1\right)\right\}\right)\left(r_{j_{0}}\right)=s_{j_{0}}+t_{j_{0}}-s_{j_{0}}=t_{j_{0}}=f(\{m(n) !\})\left(r_{j_{0}}\right) .
$$

Next, we suppose that $r$ is a prime number with $r \neq r_{j}$, for $j=1,2, \ldots, l$. In this case we divide a proof into three subcases to show that

$$
f\left(\left\{k m\left(n_{0}+1\right) \ldots m\left(n_{0}+n-1\right)\right\}\right)(r)=f(\{m(n) !\})(r) .
$$

(i) Case of $1 \leq f(\{m(n) !\})(r)<\infty$. Then $r$ does not divide $m\left(n_{0}\right)$ !. Hence there is an $n_{1} \in \mathbf{N}$ with $n_{1} \geq n_{0}+1$ such that $r^{t_{0}}$ divides $\frac{m\left(n_{1}\right) !}{m\left(n_{0}\right) !}$, where $t_{0}=f(\{m(n) !\})(r)$. Thus

$$
f\left(\left\{k m\left(n_{0}+1\right) \ldots m\left(n_{0}+n-1\right)\right\}\right)(r)=t_{0}=f(\{m(n) !\})(r) .
$$

(ii) Case of $f(\{m(n) !\})(r)=0$. Then $r$ does not divide $k$ and $m(n)$, for any $n \in \mathbf{N}$. Thus

$$
f\left(\left\{k m\left(n_{0}+1\right) \ldots m\left(n_{0}+n-1\right)\right\}\right)(r)=0=f(\{m(n) !\})(r) .
$$

(iii) Case of $f(\{m(n) !\})(r)=\infty$. Then, by Lemma 2.1, there are countably many $n \in \mathbf{N}$ with $n \geq n_{0}+1$ such that $r$ divides $m(n)$. Thus 


$$
f\left(\left\{k m\left(n_{0}+1\right) \ldots m\left(n_{0}+n-1\right)\right\}\right)(r)=\infty=f(\{m(n) !\})(r) .
$$

Therefore, since $f\left(\left\{k m\left(n_{0}+1\right) \ldots m\left(n_{0}+n-1\right)\right\}\right)=f(\{m(n) !\})$, by Glimm [6, Theorem $1.12] p A p \cong A$. Q.E.D.

TheORem 2.10. Let $n_{0}$ be a positive integer and $p$ a projection in $M_{m\left(n_{0}\right) \text { ! with }}$ $\tau(p)=\frac{k}{m\left(n_{0}\right) !}$. Let $r_{1}^{s_{1}} \ldots r_{l}^{s_{l}}$ be a factor of $m\left(n_{0}\right)$ ! with $1 \leq f(\{m(n) !\})\left(r_{j}\right)=t_{j}<\infty$, for $j=1,2 \ldots, l$, such that $r_{j}$ does not divide $\frac{m\left(n_{0}\right) !}{r_{1}^{s_{1}}, \ldots, r_{l}^{\prime l}}$ and $r$ does not divide $m\left(n_{0}\right) !$, for any prime number $r$ with $r \neq r_{j}$ for $j=1,2, \ldots, l$ and $1 \leq f(\{m(n) !\})(r)<\infty$. Then $p A p \cong A$ if and only if there is a $k_{1} \in \mathbf{N}$ such that $k=r_{1}^{s_{1}} \ldots r_{l}^{s_{l}} k_{1}$ and $k_{1}=1$ or $k_{1}=c_{1}^{d_{1}} \ldots c_{h}^{d_{h}}$ with $f(\{m(n) !\})\left(c_{j}\right)=\infty$ and $d_{j} \neq 0$, for $j=1,2, \ldots, h$.

Proof. This is immediate, by Lemmas $2.7,2.8$ and 2.9. Q.E.D.

Let $A$ be a UHF-algebra of type $\{m(n) !\}$. We suppose that

$\#\{r \mid r$ is a prime number with $1 \leq f(\{m(n) !\})(r)<\infty\}=\infty$,

$\#\{r \mid r$ is a prime number with $f(\{m(n) !\})(r)=\infty\}=0$.

In this case $k_{1}$ in the statement of Theorem 2.10 is always equal to 1 , and so $p A p \cong A$ if and only if $\tau(p)=1$. By Remark 1.5 we obtain the following theorem.

Theorem 2.11. With the assumptions above, for any automorphism $\beta$ of $A \otimes \boldsymbol{K}$, we have $\beta_{*}=$ id on $K_{0}(A \otimes K)$.

3. Examples. Let $B$ be a $C^{*}$-algebra and $M(B)$ its multiplier algebra. Let $\operatorname{Aut}(B)$ be the group of all automorphisms of $B$. For each unitary element $w \in M(B)$, let $\operatorname{Ad}(w)$ denote the automorphism of $B$ defined by $\operatorname{Ad}(w)(b)=w b w^{*}$, for any $b \in B$. We call $\operatorname{Ad}(w)$ a generalized inner automorphism of $B$, and we denote by $\operatorname{Int}(B)$ the group of all generalized inner automorphisms of $B$. It is easily seen that $\operatorname{Int}(B)$ is a normal subgroup of $\operatorname{Aut}(B)$. We note that if $B$ is unital, $\operatorname{Int}(B)$ is the group of all inner automorphisms of $B$, since $M(B)=B$. Let Pic $(B)$ be the Picard group of $B$. We note that $\operatorname{Pic}(B) \cong \operatorname{Aut}(A \otimes \mathbf{K}) / \operatorname{Int}(A \otimes \mathbf{K})$.

Let $A$ be a UHF-algebra of type $\{m(n)$ ! $\}$ and $S$ the semigroup of automorphisms of $K_{0}(A \otimes \mathbf{K})$ defined in Section 1 .

Example 3.1. We suppose that $m(n)=k \in \mathbf{N}$ with $k \geq 2$, for any $n \in \mathbf{N}$; that is, $A$ is a UHF-algebra of type $k^{\infty}$.

(1) If $k$ is a prime number, then by Theorem 2.3 we have

$$
S=\left\{\frac{1}{k^{t}} \mid t \in \mathbf{Z} \quad \text { with } \quad t \geq 0\right\} .
$$

Hence the group of automorphisms of $K_{0}(A \otimes \mathbf{K})$ induced by those of $A \otimes \mathbf{K}$ is $\left\{\frac{1}{k^{l}} \mid t \in \mathbf{Z}\right\} \cong \mathbf{Z}$. Also, by Lemma 1.1 and [8, Proposition 4], $\operatorname{Pic}(A)$ is isomorphic to a semidirect product of $\operatorname{Aut}(A) / \operatorname{Int}(A)$ with $\mathbf{Z}$. 
(2) If $k=6$, then by Theorem 2.3 we have

$$
S=\left\{\frac{2^{d_{1}} \cdot 3^{d_{2}}}{6^{t}} \mid 1 \leq 2^{d_{1}} \cdot 3^{d_{2}} \leq 6^{t}, \quad d_{1}, d_{2}, t=0,1, \ldots \quad\right\}
$$

EXAMPLE 3.2 We suppose that

$$
\begin{gathered}
\#\{r \mid r \text { is a prime number with } 1 \leq f(\{m(n) !\})(r)<\infty\}=\infty, \\
\#\{r \mid r \text { is a prime number with } f(\{m(n) !\})(r)=\infty\}=0 .
\end{gathered}
$$

Then, by Lemma 2.1, $S=\{1\}$. Hence the group of automorphisms of $K_{0}(A \otimes \mathbf{K})$ induced by those of $A \otimes \mathbf{K}$ is $\{1\}$. Therefore $\operatorname{Pic}(A) \cong \operatorname{Aut}(A) / \operatorname{Int}(A)$, by $\operatorname{Lemma} 1.1$ and $[\mathbf{8}$, Proposition 4].

Remark 3.3. Let $A$ be an AF-algebra by an inductive limit of finite dimensional $\mathrm{C}^{*}$-algebras for which the corresponding limit of $K_{0}$-groups is

$$
\cdots \longrightarrow \mathbf{Z}^{2} \stackrel{\phi_{n}}{\longrightarrow} \mathbf{Z}^{2} \longrightarrow \cdots,
$$

where each $\mathbf{Z}^{2}$ is endowed with its natural ordering and

$$
\phi_{n}=\left[\begin{array}{cc}
a_{n} & 1 \\
1 & 0
\end{array}\right]
$$

where $\left[a_{1}, a_{2}, \ldots, a_{n}, \ldots\right]$ is the continued fraction expansion of an irrational number $\theta$. Then, in the same way as in [8], we see that if $\theta$ is not quadratic, $\operatorname{Pic}(A) \cong \operatorname{Aut}(A) / \operatorname{Int}(A)$ and that if $\theta$ is quadratic, $\operatorname{Pic}(A)$ is isomorphic to a semidirect product of $\operatorname{Aut}(A) / \operatorname{Int}(A)$ with $\mathbf{Z}$.

\section{REFERENCES}

1. B. Blackadar, Traces on simple AF C*-algebras, J. Funct. Anal. 38 (1980), 156-168.

2. B. Blackadar, K-theory for operator algebras, (M. S. R. I. Publications, SpringerVerlag, 1986).

3. L. G. Brown, Stable isomorphism of hereditary subalgebra of $\mathrm{C}^{*}$-algebras, Pacific J. Math. 71 (1977), 335-348.

4. E. G. Effros and C. L. Shen, Approximately finite $\mathrm{C}^{*}$-algebras and continued fractions, Indiana Univ. Math. J. 29 (1980), 191-204.

5. G. A. Elliott, D. E. Evans and A. Kishimoto, Outer conjugacy classes of trace scaling automorphisms of stable UHF-algebras, preprint. $318-340$

6. J. Glimm, On a certain class in operator algebras, Trans. Amer. Math. Soc. 95 (1960),

7. K. Kodaka, Automorphisms of tensor products of irrational rotation $\mathrm{C}^{*}$-algebras and the $\mathrm{C}^{*}$-algebra of compact operators II, J. Operator Theory 30 (1993), 77-84.

8. K. Kodaka, Picard groups of irrational rotation $\mathrm{C}^{*}$-algebras, J. London Math. Soc. (2) 56 (1997), 179-188.

9. K. Kodaka, Full projections, equivalence bimodules and automorphisms of stable algebras of unital C*-algebras, J. Operator Theory 37 (1997), 357-369.

10. G. K. Pedersen, C*-algebras and their automorphism groups (Academic Press, 1979).

11. M. Pimsner and D. Voiculescu, Imbedding the irrational rotation $C^{*}$-algebra into an AF-algebra, J. Operator Theory 4 (1980), 201-210.

12. M. Rørdam, Classification of certain infinite simple $C^{*}$-algebras, preprint. 